\title{
Mechanism of long non-coding RNA MALAT1 in lipopolysaccharide-induced acute kidney injury is mediated by the miR-146a/NF- $\mathrm{kB}$ signaling pathway
}

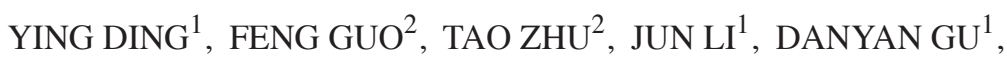 \\ WEILIANG JIANG ${ }^{1}$, YUYING LU ${ }^{2}$ and DAOYANG ZHOU ${ }^{2}$
}

\begin{abstract}
${ }^{1}$ Department of Intensive Care Unit, Sir Run Run Shaw Hospital Xiasha Campus, School of Medicine, Zhejiang University, Hangzhou, Zhejiang 310018; ${ }^{2}$ Department of Intensive Care Unit, Sir Run Run Shaw Hospital, School of Medicine, Zhejiang University, Hangzhou, Zhejiang 310016, P.R. China
\end{abstract}

Received February 9, 2017; Accepted October 19, 2017

DOI: $10.3892 /$ ijmm.2017.3232

\begin{abstract}
The present study aimed to examine the expression and function of the metastasis-associated lung adenocarcinoma transcript 1 (MALAT1)/microRNA (miR)-146a/nuclear factor (NF)- $\kappa \mathrm{B}$ axis in lipopolysaccharide (LPS)-induced acute kidney injury (AKI). The mRNA levels of MALAT1 and miR-146a in AKI tissues and cells were detected using reverse transcription-quantitative polymerase chain reaction analysis. The NF- $\kappa B$ pathway proteins and cell viability were assessed using western blot analysis and the MTT method, respectively. The secretion of inflammatory factors was determined using the ELISA method. The present study also examined effects of the abnormal expression of MALAT1 and miR-146a on cytokines and the NF- $\mathrm{KB}$ pathway. A potential binding region between MALAT1 and miR-146a was confirmed via RNA immunoprecipitation. The results revealed that the upregulation of MALAT1 reduced the expression of miR-146a, and there was a negative linear correlation between MALAT1 and miR-146a in a RNA-induced silencing complex-dependent manner. The expression levels of miR-146a were lower in the kidney injury specimens and NRK-52E cells, compared with those in the controls. MALAT1 knockdown and the overexpression of miR-146a reduced the production of phosphorylated inhibitor of NF- $\mathrm{KB}$ and np65 protein. miR-146a was found to be transcriptionally induced by $\mathrm{NF}-\mathrm{\kappa B}$, and miR-146a repressed the pro-inflammatory NF- $\kappa B$ pathway and downstream transcription factors. Taken together, these
\end{abstract}

Correspondence to: Professor Daoyang Zhou, Department of Intensive Care Unit, Sir Run Run Shaw Hospital, School of Medicine, Zhejiang University, 3 Qingchun Road, Hangzhou, Zhejiang 310016, P.R. China

E-mail: 3195023@zju.edu.cn

Key words: long non-coding RNA, metastasis-associated lung adenocarcinoma transcript 1 , microRNA-146a, nuclear factor- $\kappa \mathrm{B}$, acute kidney injury, lipopolysaccharide data indicated that the MALAT1/miR-146a/NF-кB pathway exerted key functions in LPS-induced AKI, and provided novel insights into the mechanisms of this therapeutic candidate for the treatment of the disease.

\section{Introduction}

Acute kidney injury (AKI) is a critical care syndrome in clinical practice, which is characterized by a rapid decline in renal function, and is responsible for high morbidity and mortality rates of patients in intensive care units (1). The clinical symptoms of AKI are caused by diverse components, including sepsis-induced infection (2), liver transplantation (3), cardiac surgery (4) and contrast media (5). Lipopolysaccharide (LPS) is an endotoxin produced following infection by Gram negative bacteria, and remains the most common trigger for AKI (6). In addition, LPS-induced AKI often develops in patients with decreased immune-competence, resulting in severe renal failure and exacerbated mortality rates (7). It has been suggested the onset of LPS-induced AKI is associated with an elevation of inflammatory cytokines (8) and transcription factors, for example nuclear factor $(\mathrm{NF})-\kappa B$, which consequentially induce pro-inflammatory mediators, including tumor necrosis factor- $\alpha$ (TNF- $\alpha$ ), interleukin (IL)-1 and IL-6, which coordinate the elimination of pathogens and infected cells (9). Although the pathogenic factors of AKI are clear, the complex biological and molecular mechanisms underlying AKI remain to be fully elucidated.

MicroRNAs (miRNAs; $22 \mathrm{nt}$ ) are important in gene regulation and are involved in diverse biological processes, including cell growth, migration, apoptosis and differentiation, by binding to the 3'-untranslated regions of mRNAs (10). In addition to feedback regulation at the level of transcription, miRNAs have been identified to serve in post-transcriptional negative feedback loops, which modulate inflammatory signaling (11). miR-146a was previously found to be transcriptionally induced by NF- $\mathrm{\kappa B}$ in response to the activation of innate immune signaling in monocytes (12) and inhibit activation of the NF- $\kappa B$ pathway (13). In addition, the miR-146a-induced suppression of NF- $\mathrm{kB}$-driven monocyte/macrophage activation 
and atherosclerosis are regulated by the expression of cellular apolipoprotein E (14). A defect in the NF- $\kappa \mathrm{B} / \mathrm{miR}-146 \mathrm{a}$ negative feedback loop may be involved in the pathogenesis of diabetic neuropathy (15). These previous findings demonstrate that miR-146a is involved in a negative feedback loop to control the $\mathrm{NF}-\kappa \mathrm{B}$ pathway. However, the function of the miR-146a/NF- $\kappa$ B pathway in AKI remains to be fully elucidated. Therefore, the identification of $\mathrm{miR}-146 \mathrm{a} / \mathrm{NF}-\kappa \mathrm{B}$, and the clarification of its potential mechanisms, is critical for developing efficient therapies against AKI.

Long non-coding RNAs (lncRNAs; 200 nt) are a class of RNAs, which do not encode proteins (16). Accumulating evidence shows that lncRNAs are widely expressed in various human somatic tissues and are involved in diverse cellular events, including epigenetic regulation, gene transcription, mRNA processing and gene translation (17). Compared with the well-reported dysregulated miRNAs, which are involved in various diseases, only a small number of lncRNAs have been investigated in relation to their functions in kidney cancer, including PRINS (18), CRNDE (19) and H19 (20), and even fewer have been recognized in the process of AKI. In the field of nephropathy, metastasis-associated lung adenocarcinoma transcript 1 (MALAT1) was revealed to be upregulated in clear cell renal cell carcinoma (ccRCC) and acted as a novel molecule involved in the progression of ccRCC (21). However, whether MALAT1 was associated with the malignant progression of AKI remains to be elucidated and warrants further investigations to examine the underlying mechanisms.

The present study aimed to determine the expression and function of MALAT1, miR-146a and NF- $\kappa$ B pathway factors in AKI tissues and cells. The present study also investigated the interactions among the three regulators in the regulation of AKI malignant behavior and the potential molecular pathways involved.

\section{Materials and methods}

Animals. Male Sprague-Dawley rats ( $\mathrm{n}=30$; age, 6 weeks; weight, 22-25 g) were obtained from the Laboratory Animal Center of Zhejiang University (Hangzhou, China) and were maintained in a standard laboratory in an air-conditioned room at $18-26^{\circ} \mathrm{C}$ with free access to food and water. Artificial lighting was provided on a 12/12-h light/dark cycle. All experimental protocols were performed according to the Guide for the Care and Use of Laboratory Animals and were approved by the Ethics Review Board for Animal Studies of the School of Medicine, Zhejiang University.

In vivo AKI animal model. Following adaptation to the laboratory environment for 1 week, the rats were selected for establishment of the AKI model. The animals were randomly divided into two groups ( $\mathrm{n}=15 /$ group), comprising a control and LPS-treated AKI group. The animals in the AKI model groups were administered an intraperitoneal injection of LPS $(10 \mathrm{mg} / \mathrm{kg}) 6 \mathrm{~h}$ prior to sacrifice by cervical dislocation following the inhalation of 1,1,1-trichloroethane (TCE) $(22,23)$. Blood samples were obtained and centrifuged at 3,600 x g for $10 \mathrm{~min}$ at $4^{\circ} \mathrm{C}$ to obtain serum. The blood samples were then stored at $-20^{\circ} \mathrm{C}$ and kidney samples were stored at $-80^{\circ} \mathrm{C}$ for further analysis.
Cell culture. The NRK-52E normal rat kidney epithelial cell line and HK-2 human kidney tubular epithelial cell line were purchased from the Institute of Biochemistry and Cell Biology of the Chinese Academy of Sciences (Shanghai, China). The cells were maintained in Dulbecco's modified Eagle's medium (DMEM; Invitrogen; Thermo Fisher Scientific, Inc., Waltham, MA, USA) supplemented with $10 \%$ fetal bovine serum (FBS; Invitrogen; Thermo Fisher Scientific, Inc.) and antibiotics (100 U/ml penicillin and $100 \mathrm{mg} / \mathrm{ml}$ streptomycin) in a humidified atmosphere of $5 \% \mathrm{CO}_{2}$ and $95 \% \mathrm{O}_{2}$ at $37^{\circ} \mathrm{C}$.

In vivo LPS treatment. The LPS was prepared in ultrapure water and used to produce the working dilution at a concentration of $0.5 \mu \mathrm{g} / \mathrm{ml}$ (24) in serum-free DMEM for direct application to cell cultures (25). The NRK-52E and HK-2 cells were plated in 96-well plates at a density of $5 \times 10^{4}$ cells $/ \mathrm{ml}$ per well for $24 \mathrm{~h}$, following which they were challenged with LPS and then incubated for another $24 \mathrm{~h}$. Cell viability was assessed using an MTT assay.

Cell transfection. The plasmid for the overexpression of MALAT1 was constructed by inserting the amplified MALAT1 cDNA using a MiRcute miRNA first-strand cDNA synthesis kit (Tiangen Biotech Co., Ltd., Beijing, China) according to the manufacturer's instructions into the pCDNA3.1 vector (Invitrogen; Thermo Fisher Scientific,Inc.), resulting in pcDNA MALAT1. Small interfering RNA (si)-MALAT1 targeting MALAT1 and negative control siRNA were purchased from Qiagen GmbH (Hilden, Germany). The miR-146a mimic/negative control mimic and the miR-146a inhibitor/negative control inhibitor were obtained from Guangzhou RiboBio Co., Ltd. (Guangzhou, China). For cell transfection, the cells were incubated with si-MALAT1/pcDNA MALAT1, miR-146a mimic/miR-146a inhibitor or their corresponding controls using Lipofectamine 2000 (Invitrogen; Thermo Fisher Scientific, Inc.) according to the manufacturer's instructions, and the cells were harvested $48 \mathrm{~h}$ later for subsequent experiments.

The specific primers used were as follows: human si-MALAT1 sense, GCAAAUGAAAGCUACCAAUTT and antisense, AUUGGUAGCUUUCAUUUGCTT; rat si-MALAT1 sense, GCAGUUUAGGAGAUUGUAATT and antisense, UUACAAUCUCCUAAACUGCTT; negative control siRNA sense, UUCUCCGAACGUGUCACGUTT and antisense, ACGUGACACGUUCGGAGAATT.

Western blot analysis. Total protein was extracted from the kidneys and cultured cells, and the concentrations were estimated by collecting equal number of cells and homogenizing using RIPA lysis buffer. The protein concentrations of the samples were determined using a BCA protein assay kit (Bio-Rad Laboratories, Inc., Hercules, CA, USA) and standards. The proteins $(50 \mu \mathrm{g})$ were denatured with sodium dodecyl sulfate (SDS) sample buffer, separated by $10 \%$ SDS polyacrylamide gel electrophoresis and then transferred onto PVDF membranes (EMD Millipore, Billerica, MA, USA). Following blocking with 5\% skim milk for $2 \mathrm{~h}$ at room temperature, the membranes were incubated with primary antibodies including phosphorylated inhibitor of

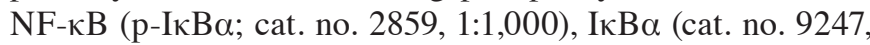


1:1,000), B-cell lymphoma 2 (Bcl-2; cat. no. 3498, 1:1,000), Bcl-2-associated X protein (Bax; cat. no. 2772, 1:1,000), and caspase-3 (cat. no. 9662, 1:1,000) (all from Cell Signaling Technology, Boston, MA, USA), np65 (cat. no. ab83063, 1:1,000; Abcam, Cambridge, MA, USA), lamin B (cat. no. $13435,1: 1,000$ ) and $\beta$-actin (cat. no. 8457, 1:1,000) (both from Cell Signaling Technology) overnight at $4^{\circ} \mathrm{C}$. Following the addition of anti-rabbit horseradish peroxidase-conjugated IgG secondary antibodies (cat. no. ab6721, 1:2000; Abcam) for $2 \mathrm{~h}$ at room temperature. The protein bands on the membranes were detected using an enhanced chemiluminescence system (UVP, Inc., Upland, CA, USA). The intensity values of the bands were adjusted relative to the expression of the internal reference $(\beta$-actin and lamin $\mathrm{B})$.

$R N A$ extraction and reverse transcription-quantitative polymerase chain reaction ( $R T-q P C R)$ analysis. Total RNA from the tissues and cells was extracted using TRIzol (Thermo Fisher Scientific, Inc.) according to the manufacturer's instructions. The mRNA and miRNA were polyadenylated using a poly-A polymerase-based First-Strand Synthesis kit (Takara Biotechnology Co., Ltd., Shanghai, China) and reverse transcription of the total mRNA was performed using a PrimeScript RT reagent kit according to the manufacturer's instructions. The reactions were incubated in a 96 -well plate at $95^{\circ} \mathrm{C}$ for $10 \mathrm{~min}$, followed by 40 cycles of $95^{\circ} \mathrm{C}$ for $15 \mathrm{sec}, 60^{\circ} \mathrm{C}$ for $30 \mathrm{sec}$ and $72^{\circ} \mathrm{C}$ for $30 \mathrm{sec}$. The resulting amplification were quantified on an ABI 7500HT system (Applied Biosystems; Thermo Fisher Scientific, Inc.) using SYBR-Green I. U6 or GAPDH were used as endogenous controls. Relative fold expression levels were calculated with the comparative quantification cycle $\left(2^{-\Delta \Delta \mathrm{Cq}}\right)$ method (26).

The primer sequences were listed as follows: miRNA-146a forward, 5'-ACACTCCAGCTGGGTGAGAACTGAATT CCA-3' and reverse, 5'-CTCAACTGGTGTCGTGGAGTCG GCAATTCAGTTGAGAACCCATG-3'; and MALAT1 forward, 5'-GCGACGAGTTGTGCTGCTATCT-3' and reverse, 5'-ACACTGCTCTGGGTCTGCTTTT-3'.

Assessment of biochemical parameters. Serum levels of blood urea nitrogen (BUN; mmol/l) and creatinine $(\mathrm{Cr} ; \mu \mathrm{mol} / \mathrm{l})$ were detected using specific kits according to the manufacturer's instructions. The serum expression levels of TNF- $\alpha$ and IL-6 (ng/l) were determined using commercial ELISA kits according to the manufacturer's instructions. All assays were performed in triplicate.

Cell viability assay. Cell proliferation was measured using an MTT assay. The cells were seeded in a 96-well plate and cultured in normal medium. Following transfection, the cells were incubated in $0.1 \mathrm{mg} / \mathrm{ml} \mathrm{MTT}$ at $37^{\circ} \mathrm{C}$ for $4 \mathrm{~h}$ and lysed in dimethyl sulfoxide at room temperature for $10 \mathrm{~min}$. The absorbance in each well was measured on a microplate reader at a wavelength of $490 \mathrm{~nm}$ (Bio-Rad Laboratories, Inc.).

RNA immunoprecipitation (RIP). LncBase Predicted v.2 bioinformatics tools (http://carolina.imis.athena-innovation. $\mathrm{gr} /$ ) was used to predict the potential interaction of lncRNA MALAT1 and miR-146a. The cells were co-transfected with a pcDNA-MS2/pcDNA-MALAT1-MS2 vector and
miR-146a mimic or non-targeting miRNA mimic. At $48 \mathrm{~h}$ post-transfection, the cells were washed and lysed in radioimmunoprecipitation buffer containing $10 \%$ proteinase inhibitor cocktail and $1 \mathrm{mM}$ phenylmethylsulfonyl fluoride. A fraction of the whole cell lysate was used for RNA isolation, and the remaining lysate was subjected to IP using an antibody against immunoglobulin G (cat. no. 5873, 1:20; Cell Signaling Technology) for $18 \mathrm{~h}$ at $4^{\circ} \mathrm{C}$. The RNA from the whole cell lysates and the RIP fractions were extracted with TRIzol according to the manufacturer's instructions. The relative mRNA expression levels of MALAT1 and miR-146a were determined using RT-qPCR analysis, as described above. The relative mRNA enrichment in the RIP fractions was computed based on the ratio of relative mRNA levels in the RIP fractions and the relative mRNA levels in the whole cell lysates (input).

Statistical analysis. All data are expressed as the mean \pm standard deviation and were analyzed using SPSS 16.0 software for Windows (SPSS, Inc., Chicago, IL, USA). An independent sample t-test was used to determine the statistical differences between two groups. Multiple group comparisons were tested using one-way analysis of variance (ANOVA). Pearson's correlation analysis was used to assess the correlation between MALAT1 and miR-146a. $\mathrm{P}<0.05$ was considered to indicate a statistically significant difference.

\section{Results}

Expression of MALAT1 and miR-146a are inversely correlated in the LPS-induced AKI model. To examine aberrant gene expression in response to kidney injury, a rat AKI model was successfully established. Serum and infarcted kidney tissues were isolated for subsequent analysis using RT-qPCR and western blot analyses. The results showed a significant several-fold increase in serum level of BUN in the AKI group, compared with that in the normal control (Fig. 1A), in line with markedly elevated $\mathrm{Cr}$ production (Fig. 1B). Concomitantly, the ELISA results revealed that LPS significantly enhanced the expression of serum inflammatory factors IL-6 and TNF- $\alpha$ (Fig. 1C and D). In addition, the RT-qPCR analysis indicated that the relative expression level of miR-146a was decreased following LPS treatment, compared with that in the control group (Fig. 1E), whereas the expression of MALAT1 was upregulated in the kidneys stimulated by LPS $(0.5 \mu \mathrm{g} / \mathrm{ml}$; Fig. 1F). To further confirm the role of MALAT1 in the AKI rats, the potential correlation between MALAT1 and miR-146a was examined using Pearson's correlation analysis. The expression of MALAT1 negatively correlated with the expression of miR-146a ( $\mathrm{P}=0.0219 ; \mathrm{r}=-0.7084$; Fig. $1 \mathrm{G})$. The results of the western blot analysis for the protein expression levels of p-IкB $\alpha, n p 65$ and apoptosis-associated regulators showed that the production of $\mathrm{p}-\mathrm{I} \kappa \mathrm{B} \alpha, \mathrm{np} 65$, cleaved (c)-caspase- 3 and Bax was increased in the model group, compared with the control group, which was in contrast to the level of Bcl-2 in the kidney tissues (Fig. 1H). These results indicated that the NF- $\kappa \mathrm{B}$ np65-mediated pathways affected the LPS-induced upregulation of MALAT1 and downregulation of miR-146a.

MALAT1 silencing protects NRK-52E kidney cells from LPS challenge. To determine the abnormal factors expressed in 

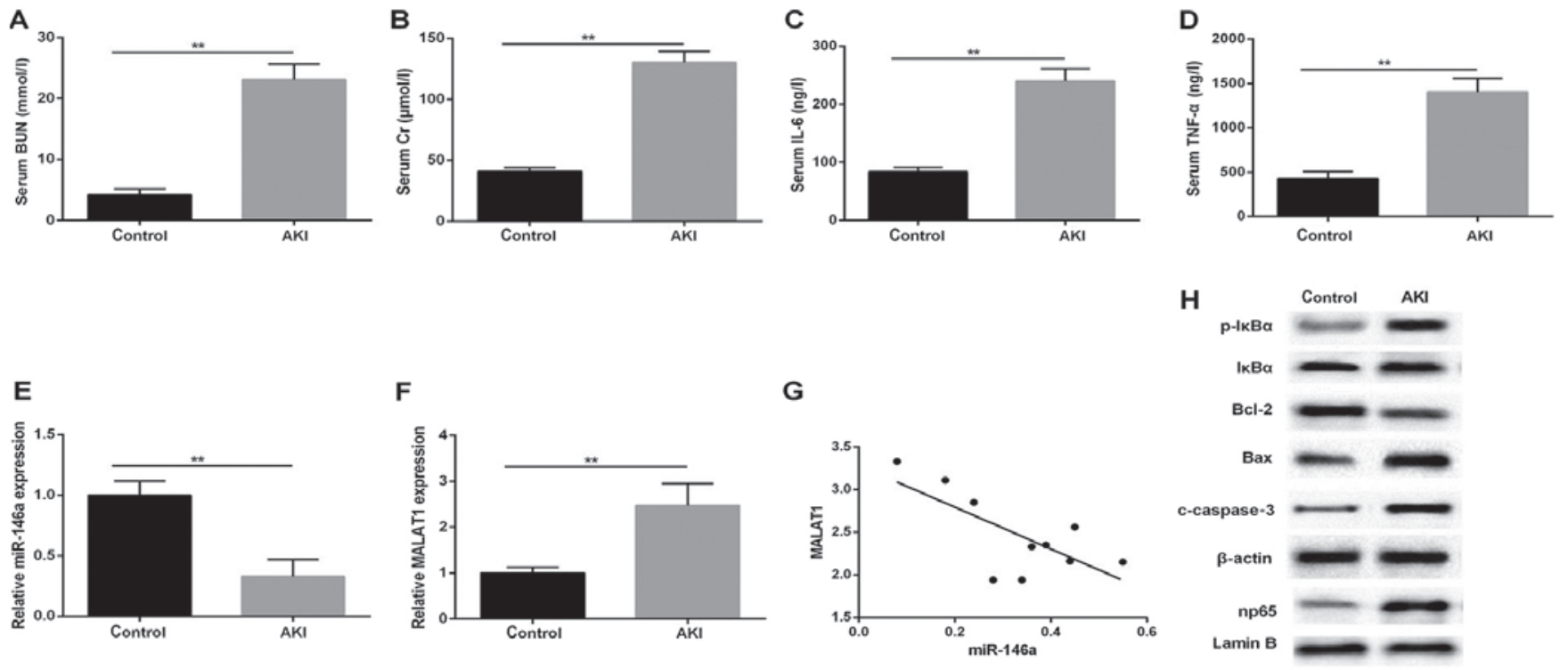

Figure 1. MALAT1 expression negatively correlates with the expression of miR-146a in a LPS-induced AKI rat model. Serum levels of (A) BUN (mmol/1), (B) $\mathrm{Cr}(\mu \mathrm{mol} / \mathrm{l})$, (C) IL-6 and (D) TNF- $\alpha$ (ng/l). Reverse transcription-quantitative polymerase chain reaction analysis of the expression of (E) miR-146a and (F) MALAT1 in rat AKI tissues (n=15 for each group); U6 and GAPDH were used as internal controls, respectively. (G) Correlation analysis between MALAT1 and miR-146a ( $\mathrm{P}=0.0219 ; \mathrm{r}=0.7084)$. (H) Western blot analysis of $\mathrm{p}-\mathrm{I} \kappa \mathrm{B} \alpha, \mathrm{np} 65$, c-caspase-3, Bax and $\mathrm{Bcl}-2$ in tissues. $\beta$-actin was used as an internal control. ${ }^{* *} \mathrm{P}<0.01$ vs. control group. All data are expressed as the mean \pm standard deviation of at least three independent experiments. MALAT1, metastasis-associated lung adenocarcinoma transcript 1; LPS, lipopolysaccharide; AKI, acute kidney injury; miR, microRNA; IL-6, interleukin-6; TNF- $\alpha$, tumor necrosis factor- $\alpha$; BUN, blood urea nitrogen; $\mathrm{Cr}$, creatinine; $\mathrm{p}$-IкB $\alpha$, phosphorylated inhibitor of nuclear factor-кB; Bcl-2, B-cell lymphoma 2; Bax, Bcl-2-associated X protein.

\section{NRK-52E}

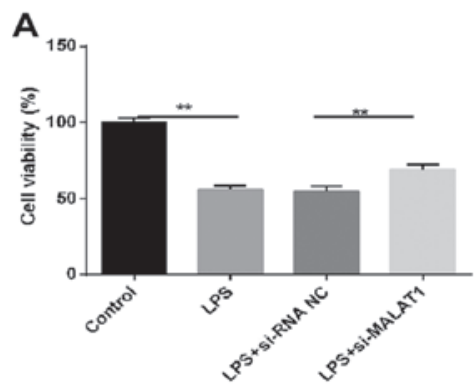

D

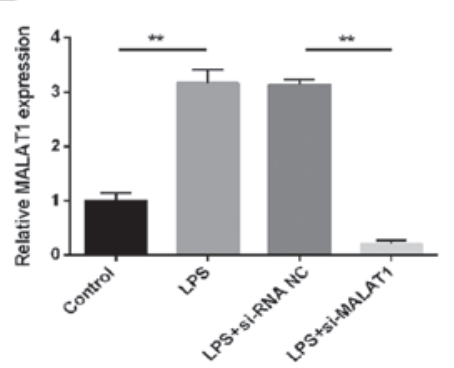

B

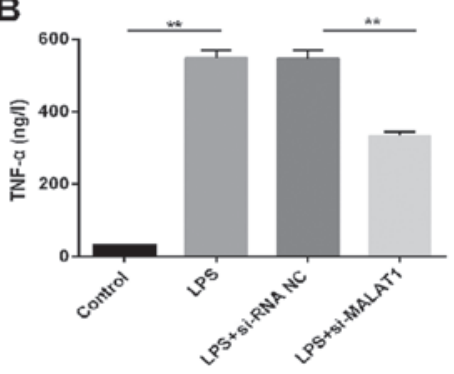

E

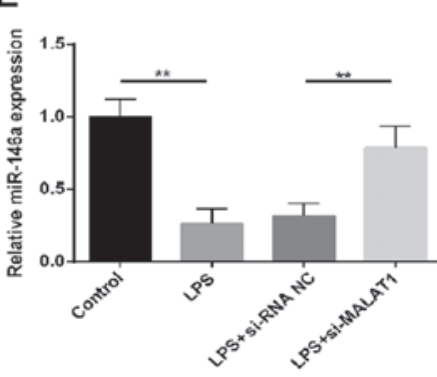

C
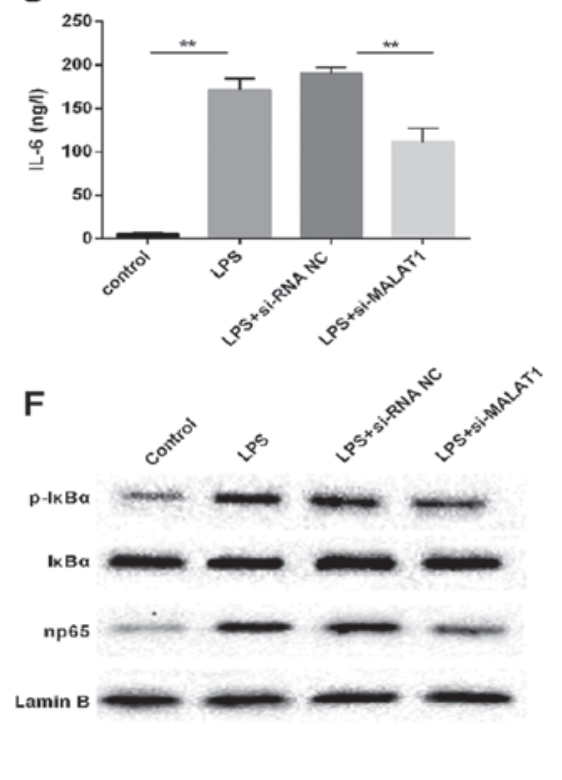

Figure 2. MALAT1 silencing protects NRK-52E renal tubular epithelial cells from LPS challenge. In vitro, cells were randomly divided into four groups: Control, LPS, LPS+si-RNA NC and LPS+si-MALAT1. (A) Cell viability was assessed using the MTT method. Expression levels of (B) TNF- $\alpha$ and (C) IL-6 (ng/l). Reverse transcription-quantitative polymerase chain reaction analysis of the expression of (D) MALAT1 and (E) miR-146a in cells. U6 and GAPDH were used as internal controls, respectively. (F) Western blot analysis of protein levels of NF- $\mathrm{kB}$ regulatory factors in NRK-52E cells. Lamin B was used as an internal control. ${ }^{* *} \mathrm{P}<0.01$ between groups. All data are expressed as the mean \pm standard deviation of at least three independent experiments. MALAT1, metastasis-associated lung adenocarcinoma transcript 1; miR, microRNA; LPS, lipopolysaccharide; si, small interfering RNA; NC, negative

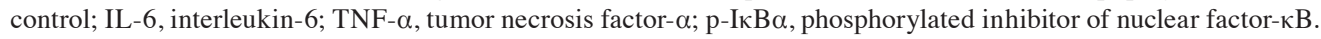

LPS-injured kidney epithelial cells, NRK-52E and HK-2 cells were used for investigation of the mechanism. The NRK-52E cells were subjected to LPS treatment, simulating the LPS-induced AKI model in vitro. Cell viability (Fig. 2A) was detected, and changes in the expression of MALAT1, miR-146a and np65, and the production of TNF- $\alpha$ in the supernatant were analyzed. Similar results were observed in the LPS-treated NRK-52E renal tubular epithelial 


\section{NRK-52E}

A

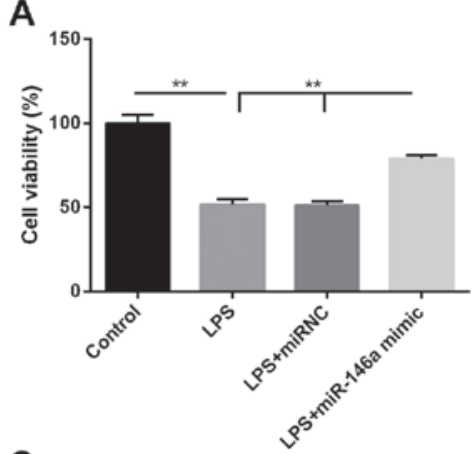

C

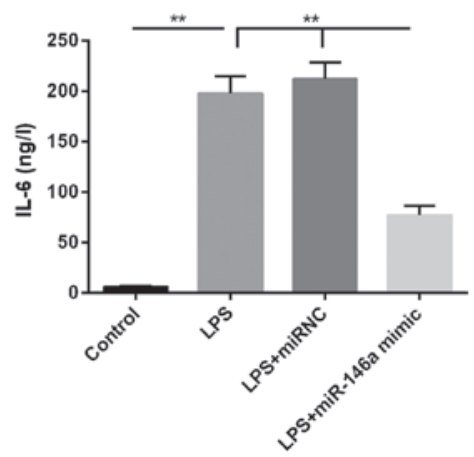

B

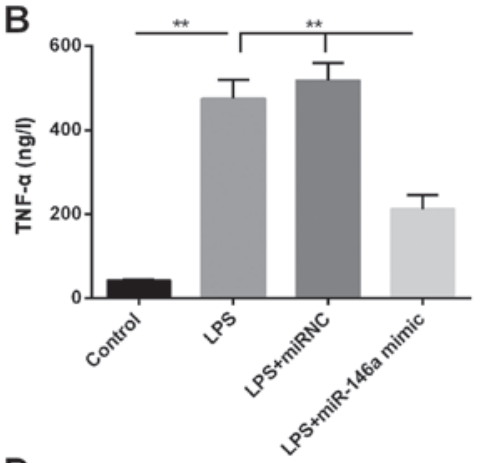

D

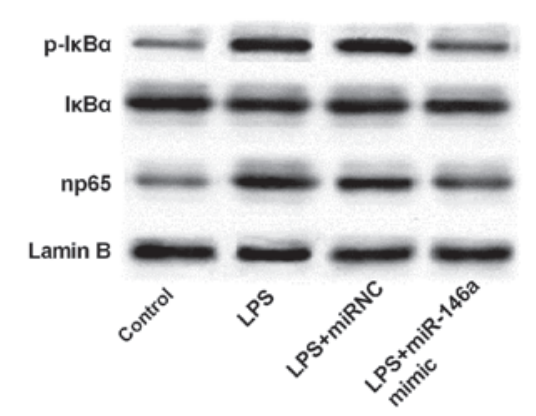

Figure 3. miR-146a attenuates the LPS-induced expression of NF-кB pathway-associated molecules. In vitro, cells were randomly divided into four groups: Control, LPS, LPS+miR NC and LPS+miR-146a mimic. (A) Cell viability was assessed using the MTT method. Expression of (B) TNF- $\alpha$, (C) IL-6 (ng/l) and (D) NF-kB regulatory proteins were examined in NRK-52E cells. Lamin B was used as an internal control. ${ }^{* *} \mathrm{P}<0.01$ between groups. All data are expressed as the mean \pm standard deviation of at least three independent experiments. miR, microRNA; LPS, lipopolysaccharide; NC, negative control; NF- $\mathrm{kB}$, nuclear

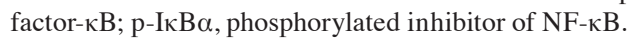

cells, including increased production of TNF- $\alpha$, IL-6 and MALAT1 (Fig. 2B-D). By contrast, cell viability was inhibited and the expression of miR-146a was markedly downregulated in the LPS treatment group, compared with that in the control cells (Fig. 2E). Simultaneously, the production of p-IкB $\alpha$ and np65 at the protein level were assessed using western blot analysis. As shown in Fig. 2F, the levels of NF- $\mathrm{BB}$ signaling pathway-associated molecules $\mathrm{p}$-IкB $\alpha$ and np65 were higher in the NRK-52E cells induced by LPS, compared with those in the controls. However, the above-mentioned results were reversed by si-MALAT1 in the NRK-52E cells. These results indicated that MALAT1 may be important in LPS-induced AKI, involve the LPS-induced production of TNF- $\alpha$ via NF- $\mathrm{KB}$ np65-mediated pathways.

miR-146a attenuates LPS-induced expression of NF- $\kappa B$ pathway-associated molecules. As the expression of miR-146a was downregulated following LPS treatment, the overexpression of miR-146a was enforced by transfecting the miR-146a mimic into NRK-52E cells. As shown in Fig. 3A, the LPS-induced downregulation in cell viability was reversed by the miR-146a mimic, to close to the baseline level. Further analysis of the levels of pro-inflammatory cytokines in the supernatant showed that the LPS-induced production of TNF- $\alpha$ and IL- 6 were significantly attenuated in the miR-146a-overexpressing kidney epithelial cells (Fig. 3B and C). Subsequently, the expression of NF- $\kappa B$ pathway-associated regulators ( $\mathrm{p}-\mathrm{I} \kappa \mathrm{B} \alpha$ and $\mathrm{np} 65)$ were examined using western blot analysis, and it was found that the basal levels of these proteins were suppressed in the cells transfected with miR-146a mimic, compared with those in the LPS, and LPS+miR-NC groups (Fig. 3D). Therefore, the miR-146a-mediated expression of NF- $\kappa$ B and np65 pathway members may be the underlying LPS-induced mechanism during the pathogenesis of AKI.

Protective effect of MALAT1-silencing on LPS-treated NRK-52E cells is reversed by miR-146a inhibitor. To further detect the effect of MALAT1 and miR-146a on inflammatory response-induced kidney injury, the NRK-52E cells treated with LPS were transfected with si-MALAT1 or siRNA NC, miR-146a inhibitor or miR-NC. As shown in Fig. 4A, the LPS-induced increase of cell viability was markedly attenuated in the miR-146a-inhibited NRK-52E cells. The expression levels of TNF- $\alpha$ and IL- 6 were markedly upregulated in cells co-transfected with miR-146a inhibitor and si-MALAT1, compared with those in the corresponding control group (Fig. 4B and C). The western blot analysis showed that the levels of $\mathrm{p}$-IкB $\alpha$ and np65 were promoted by the inhibition of miR-146a, compared with that in the control (Fig. 4D). Therefore, the cytoprotective effect induced by si-MALAT1 was undermined by miR-146a-inhibition.

MALAT1 is a direct target of miR-146a. There is increasing evidence that lncRNAs may be competing endogenous RNAs or molecular sponges in downregulating the expression and biological functions of miRNAs. Using a bioinformatics database (starBase v2.0), the present study determined 


\section{NRK-52E}

A

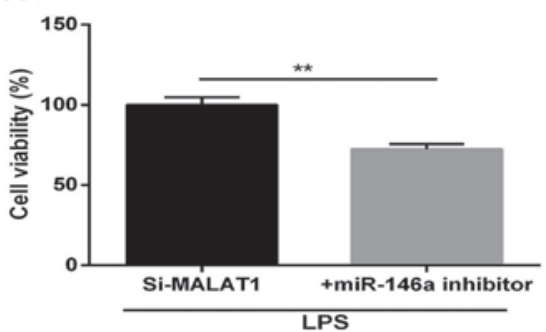

C

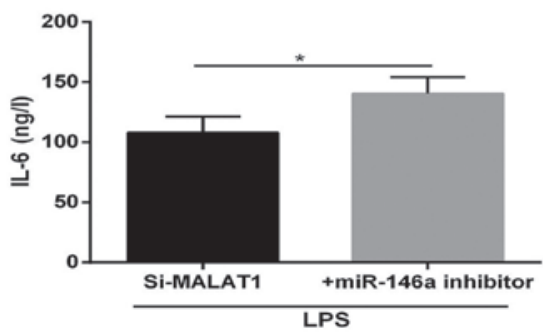

B

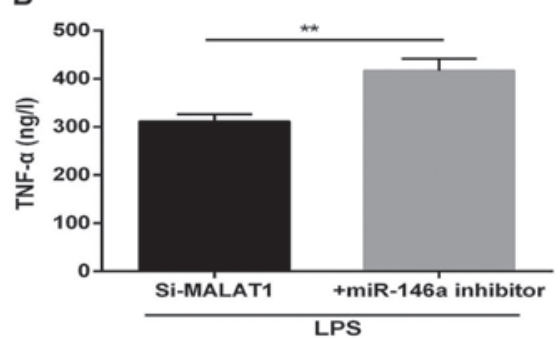

D

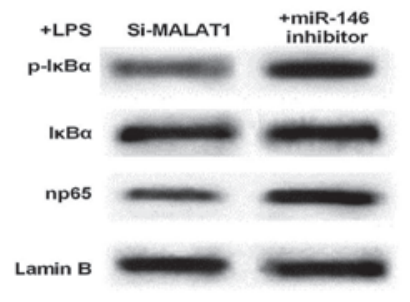

Figure 4. Cytoprotective effects of si-MALAT1 on LPS-treated acute kidney injury is reversed by the miR-146a inhibitor. Following LPS treatment, the cells were divided into si-MALAT1 and si-MALAT1+miR-146a inhibitor groups. (A) Cell viability. Expression of (B) TNF- $\alpha$, (C) IL-6 (ng/l) and (D) NF- $\kappa$ B regulatory proteins in NRK-52E cells. Lamin B was used as an internal control. ${ }^{*} \mathrm{P}<0.05$ and ${ }^{* *} \mathrm{P}<0.01$ between groups. All data are expressed as the mean \pm standard deviation of at least three independent experiments. MALAT1, metastasis-associated lung adenocarcinoma transcript 1; miR, microRNA; LPS, lipopolysaccharide; si, small interfering RNA; IL-6, interleukin-6; TNF- $\alpha$, tumor necrosis factor- $\alpha$; NF- $\kappa \mathrm{B}$, nuclear factor- $\kappa \mathrm{B}$; $\mathrm{p}-\mathrm{I} \kappa \mathrm{B} \alpha$, phosphorylated inhibitor of NF- $\kappa \mathrm{B}$.

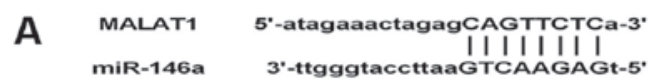

B

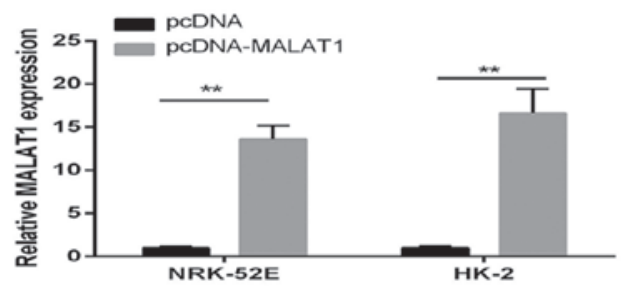

D

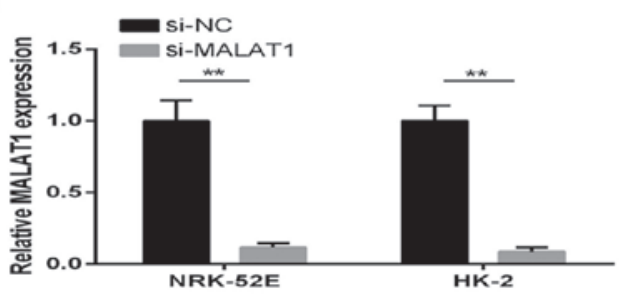

$\mathbf{F}$

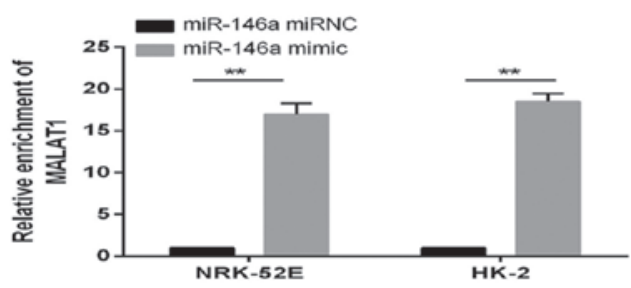

C

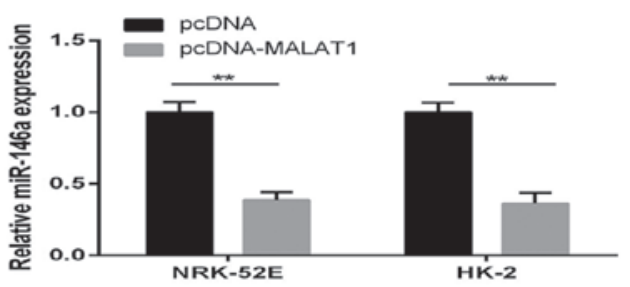

E

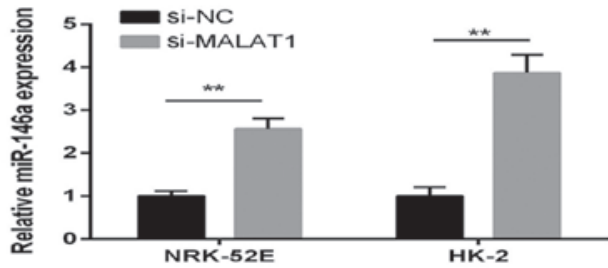

G

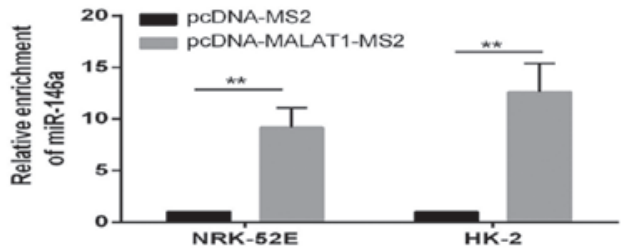

Figure 5. MALAT1 is a direct target of miR-146a. (A) MALAT1 harbors one putative binding site for miR-146a. Expression of (B) MALAT1 and (C) miR-146a in NRK-52E and HK-2 cells transfected with pcDNA-MALAT1. Expression of (D) MALAT1 and (E) miR-146a in NRK-52E and HK-2 cells transfected with si-MALAT1. GAPDH was used as the internal control. Relative expression of miR-146a in NRK-52E and HK-2 cells transfected with (C) pcDNA-MALAT1 or (E) si-MALAT1. U6 was used as the internal control. (F) Relative enrichment of MALAT1 in NRK-52E and HK-2 cells transfected with miR-146a mimic. (G) Relative enrichment of miR-146a in NRK-52E and HK-2 cells transfected with pcDNA-MALAT1-MS2. ${ }^{* *} \mathrm{P}<0.01$ between groups. All data are expressed as the mean \pm standard deviation of at least three independent experiments. MALAT1, metastasis-associated lung adenocarcinoma transcript 1; miR, microRNA; si, small interfering RNA; NC, negative control. 

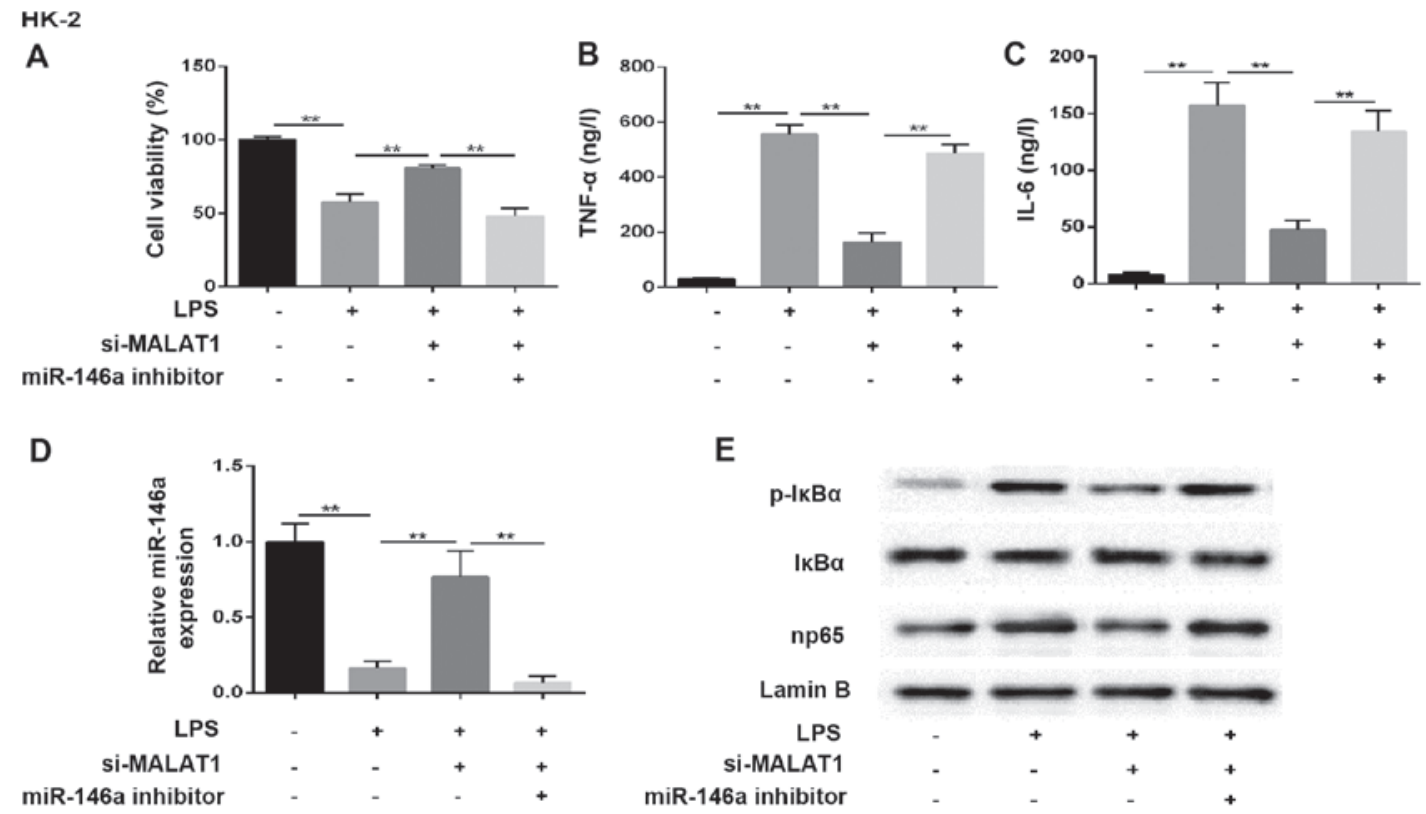

Figure 6. MALAT1 regulates the NF-kB pathway by affecting the expression of miR-146a. HK-2 cells were randomly divided into four groups: control, LPS, LPS+si-MALAT1 and LPS+si-MALAT1+miR-146a inhibitor. (A) Cell viability. Expression of (B) TNF- $\alpha$ and (C) IL-6 (ng/l). (D) Reverse transcription-quantitative polymerase chain reaction analysis of miR-146a. U6 was used as the internal control. (E) Expression of NF-kB regulatory proteins in HK-2 cells. Lamin B was used as an internal control. ${ }^{* * *} \mathrm{P}<0.01$ between groups. All data are expressed as the mean \pm standard deviation of at least three independent experiments. MALAT1, metastasis-associated lung adenocarcinoma transcript 1; miR, microRNA; LPS, lipopolysaccharide; si, small interfering RNA;

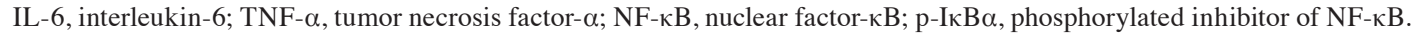

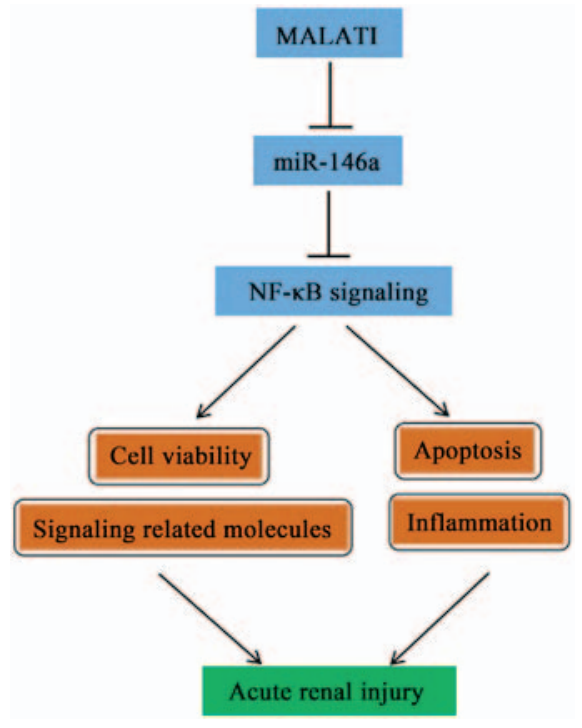

Figure 7. Diagram of the regulation mechanism of MALAT1 in the progression of AKI. MALAT1, metastasis-associated lung adenocarcinoma transcript 1; AKI, acute kidney injury; miR, microRNA; NF-kB, nuclear factor-kB.

that MALAT1 harbored one putative binding site for miR-146a (Fig. 5A). To confirm the hypothesis that miR-146a directly binds to MALAT1, the expression of miR-146a in pcDNA-MALAT1 cells was measured using RT-qPCR analysis. The expression of MALAT1 was confirmed to be significantly upregulated in NRK-52E and HK-2 cells transfected with pcDNA-MALAT1; whereas the expression of miR-146a was decreased in the pcDNA-MALAT1 group (Fig. 5B and C). By contrast, the expression of MALAT1 and miR-146a showed the opposite results when the cells were treated with si-MALAT1, compared with those in the si-NC control group (Fig. 5D and E).

An RIP assay was performed to determine whether MALAT1 and miR-146a were in the expected RNA-induced silencing complex (RISC). The RNA levels were examined using RT-qPCR analysis, which revealed significant relative enrichment of mRNA levels of MALAT1 and miR-146a, compared with those in the control group, respectively (Fig. 5F and G). Therefore, MALAT1 inhibition was confirmed to restore the expression of miR-146a in a RISCdependent manner, and there was a negative linear correlation between MALAT1 and miR-146a.

MALAT1 regulates the $N F-\kappa B$ pathway via affecting the expression of miR-146a. The mechanism underlying the effect of MALAT1 in AKI mediated by the miR-146a/NF- $\mathrm{B}$ signaling pathway was assessed in NRK-52E rat renal tubular epithelial cells, and HK-2 human renal tubular epithelial cells were used to confirm the findings. In cells co-treated with LPS and si-MALAT1, cell survival rate increased and the secretion of inflammatory factors decreased, compared with the LPS-only induction group. However, this effect was eliminated by miR-146a inhibitor (Fig. 6A-C). The change in the expression of miR-146a in the supernatant was analyzed accordingly, and similar results were observed in the treated groups (Fig. 6D). In addition, western blot analysis of the expression of $\mathrm{p}-\mathrm{I} \kappa \mathrm{B} \alpha$ and np65 showed that these two proteins were increased in the LPS+si-MALAT1+miR-146a inhibitor group, compared with those in cells transfected with LPS+si-MALAT1 (Fig. 6E). These results indicated that MALAT1 regulated kidney injury through the miR$146 \mathrm{a} / \mathrm{NF}-\mathrm{\kappa B}$ signaling pathway in HK-2 cells. A diagram of the mechanism is shown in Fig. 7. 


\section{Discussion}

In the present study, it was demonstrated that MALAT1 was upregulated in damaged tissues and renal tubularepithelial cells. The inhibition of MALAT1 impaired the malignant behavior of AKI and attenuated the expression of np65. By contrast, the expression of miR-146a was downregulated in renal tissue samples and cell lines. The restoration of miR-146a suppressed the cell inflammatory response, promoted cell proliferation and reduced the expression of np65. miR-146a was also found to bind to MALAT1 in a sequence-specific manner, and there was reciprocal repression between miR-146a and MALAT1, possibly induced by the RISC. NF- $\kappa$ B was identified as an important downstream regulatory factor of miR-146a and was involved in the malignant progression of AKI. NF- $\kappa \mathrm{B}$ was also confirmed to promote the adverse effects of AKI by enhancing the expression of cell inflammatory factors and promoting apoptosis.

AKI is a complex disorder for which currently there is no accepted definition; it is typically characterized by kidney function detriment, resulting in profound effects on patient health (27). Several factors can cause AKI, including predominantly acute hypoxia, trauma, and toxic or septic insults (28). Notably, LPS also induces the pathogenesis of AKI and leads to kidney failure and multi-organ damage; however, the pathogenesis of human AKI is complex and only partially elucidated (29). Therefore, it is important to examine the potential mechanism and identify effective therapeutic strategies for LPS-induced AKI.

miRNAs are vital in several biological processes through mRNA degradation or translational suppression (30). miR-146a is widely expressed in kidney diseases, and its urinary excretion is specifically associated with the development of interstitial lesions and correlated with inflammatory cell infiltration (31). Cheng et al demonstrated that the anti-inflammatory activity of miR-146a is mediated by the suppression of pro-inflammatory transcription factor NF- $\kappa \mathrm{B}$ (11). In the present study, it was found that the levels of miR-146a were markedly decreased in vitro, accompanied by increased protein levels of np65. Therefore, miR-146a may be a potential target in the process of LPS-induced AKI.

There is substantial evidence that lncRNAs are aberrantly expressed in various diseases, and are important regulators of physiological and pathological responses. Previous studies have indicated that they lncRNAs act as miRNA sponges or miRNA inhibitors (antagomirs), which interact with miRNAs and regulate the expression of miRNA target genes (32). For example, the overexpression of miR21 partly abrogated the cancer susceptibility 2-mediated inhibition of human renal cell carcinoma (RCC) 786-O and A498 cell proliferation and migration (33). The expression of HOTAIR was found to be inversely correlated with that of miR-141 in RCC, in which miR-141 bound to HOTAIR in a sequence-specific manner, and suppressed the expression and functions of HOTAIR, including proliferation and invasion (34). Cardiac hypertrophy-related factor was found to directly bind to miR-489 and regulate the expression of myeloid differentiation primary response 88 in cardiac hypertrophy (35). The present study investigated whether MALAT1 had similar effects on the levels of miR-146a in LPS-induced AKI. MALAT1 is present at high levels in several human cell types and is conserved across several mammalian species, indicating its functional importance (36). In the present study, MALAT1 was significantly increased in tissues and cell lines, and the inhibitory effect of MALAT1 on miR-146a levels was observed in NRK-52E and HK-2 renal tubular epithelial cells. The downregulation of MALAT1 mitigated cytokine secretion and the immune response, increased cell viability and increased the expression of miR-146a. These data indicated that MALAT1 may be directly or indirectly involved in inflammation and the progression of AKI. It was also confirmed that the inhibition of miR-146a reversed the promoting effects of si-MALAT1 on np65 and $\mathrm{p}-\mathrm{I} \kappa \mathrm{B} \alpha$. The knockdown of MALAT1 promoted the functions of miR-146a. These results revealed that MALAT1 was involved in LPS-induced AKI through regulating the expression and function of miR-146a. However, the exact mechanism by which MALAT1 regulates miR-146a remains to be elucidated and warrants further investigation.

In conclusion, based on the results of the present study, a simplified pathway to describe the possible involvement of MALAT1/miR-146a/NF- $\kappa$ B signaling in LPS-induced AKI was demonstrated. The findings not only provide novel insight for clarifying the complex molecular mechanisms of specific miRNAs and lncRNAs in LPS-induced AKI, but also assist in facilitating the development of miRNA- and lncRNA-directed therapeutic strategies for kidney injury.

\section{Acknowledgements}

This study was supported by the Hangzhou Health Science and Technology Plan Project (grant no. 2017A71).

\section{References}

1. Segev G, Langston C, Takada K, Kass PH and Cowgill LD: Validation of a clinical scoring system for outcome prediction in dogs with acute kidney injury managed by hemodialysis. J Vet Intern Med 30: 803-807, 2016.

2. Angeli P, Tonon M, Pilutti C, Morando F and Piano S: Sepsis-induced acute kidney injury in patients with cirrhosis. Hepatol Int 10: 115-123, 2016.

3. Linkermann A: Nonapoptotic cell death in acute kidney injury and transplantation. Kidney Int 89: 46-57, 2016.

4. Pistolesi V, Di Napoli A, Fiaccadori E, Zeppilli L, Polistena F, Sacco MI, Regolisti G, Tritapepe L, Pierucci A and Morabito S: Severe acute kidney injury following cardiac surgery: short-term outcomes in patients undergoing continuous renal replacement therapy (CRRT). J Nephrol 29: 229-239, 2016.

5. Chalikias G, Drosos I and Tziakas DN: Prevention of contrast-induced acute kidney injury: an update. Cardiovasc Drugs Ther 30: 515-524, 2016.

6. Xu D, Chen M, Ren X, Ren X and Wu Y: Leonurine ameliorates LPS-induced acute kidney injury via suppressing ROS-mediated $\mathrm{NF}-\kappa \mathrm{B}$ signaling pathway. Fitoterapia 97: 148-155, 2014.

7. Kawara M, Matsunaga R, Yamamoto Y, Yoneda G, Fujino R, Nishi K, Jono H and Saito H: Nephropreventive effect of shikonin on murine LPS-induced septic acute kidney injury via Nrf2 activation with antioxidative responses. J Clin Exp Nephrol 1: 19, 2016.

8. Heemskerk S, Masereeuw R, Russel FG and Pickkers P: Selective iNOS inhibition for the treatment of sepsis-induced acute kidney injury. Nat Rev Nephrol 5: 629-640, 2009.

9. Zhao G, Su Z, Song D, Mao Y and Mao X: The long noncoding RNA MALAT1 regulates the lipopolysaccharide-induced inflammatory response through its interaction with NF- $\kappa B$. FEBS Lett 590: 2884-2895, 2016.

10. Frixa T, Donzelli S and Blandino G: Oncogenic microRNAs: key players in malignant transformation. Cancers (Basel) 7: 2466-2485, 2015. 
11. Cheng HS, Sivachandran N, Lau A, Boudreau E, Zhao JL, Baltimore D, Delgado-Olguin P, Cybulsky MI and Fish JE: MicroRNA-146 represses endothelial activation by inhibiting pro-inflammatory pathways. EMBO Mol Med 5: 1017-1034, 2013.

12. Taganov KD, Boldin MP, Chang KJ and Baltimore D: NF-kappaB-dependent induction of microRNA miR-146, an inhibitor targeted to signaling proteins of innate immune responses. Proc Natl Acad Sci USA 103: 12481-12486, 2006.

13. Zhao JL, Rao DS, Boldin MP, Taganov KD, O'Connell RM and Baltimore D: NF-kappaB dysregulation in microRNA-146a-deficient mice drives the development of myeloid malignancies Proc Natl Acad Sci USA 108: 9184-9189, 2011.

14. Li K, Ching D, Luk FS and Raffai R: Abstract 155: MicroRNA-146a suppression of NF- $\kappa B$-driven monocyte/macrophage activation and atherosclerosis is regulated by cellular ApoE expression. Arterioscler Thromb Vasc Biol 35: A155, 2015.

15. Yousefzadeh N, Alipour MR and Soufi FG: Deregulation of NF- $\mathrm{B}$-miR-146a negative feedback loop may be involved in the pathogenesis of diabetic neuropathy. J Physiol Biochem 71: 51-58, 2015.

16. Huang YA, Chen X, You ZH, Huang DS and Chan KC: ILNCSIM: improved lncRNA functional similarity calculation model. Oncotarget 7: 25902-25914, 2016

17. Gong W, Zheng J, Liu X, Ma J, Liu Y and Xue Y: Knockdown of NEAT1 restrained the malignant progression of glioma stem cells by activating microRNA let-7e. Oncotarget 7: 62208-62223, 2016.

18. Yu TM, Palanisamy K, Sun KT, Day YJ, Shu KH, Wang IK, Shyu WC, Chen P, Chen YL and Li CY: RANTES mediates kidney ischemia reperfusion injury through a possible role of HIF-1 $\alpha$ and lncRNA PRINS. Sci Rep 6: 18424, 2016.

19. Shao K, Shi T, Yang Y, Wang X, Xu D and Zhou P: Highly expressed lncRNA CRNDE promotes cell proliferation through $\mathrm{Wnt} / \beta$-catenin signaling in renal cell carcinoma. Tumour Biol: Oct 6, 2016 (Epub ahead of print).

20. Wang L, Cai Y, Zhao X, Jia X, Zhang J, Liu J, Zhen H, Wang T, Tang X, Liu Y, et al: Down-regulated long non-coding RNA H19 inhibits carcinogenesis of renal cell carcinoma. Neoplasma 62 : 412-418, 2015

21. Zhang HM, Yang FQ, Chen SJ, Che J and Zheng JH: Upregulation of long non-coding RNA MALAT1 correlates with tumor progression and poor prognosis in clear cell renal cell carcinoma. Tumour Biol 36: 2947-2955, 2015

22. Nair AR, Masson GS, Ebenezer PJ, Del Piero F and Francis J: Role of TLR4 in lipopolysaccharide-induced acute kidney injury: protection by blueberry. Free Radic Biol Med 71: 16-25, 2014.

23. Shin S, Kim Y, Jeong S, Hong S, Kim I, Lee W and Choi S: The therapeutic effect of human adult stem cells derived from adipose tissue in endotoxemic rat model. Int J Med Sci 10: 8-18, 2013.
24. Qi M, Yin L, Xu L, Tao X, Qi Y, Han X, Wang C, Xu Y, Sun H, Liu K, et al: Dioscin alleviates lipopolysaccharideinduced inflammatory kidney injury via the microRNA let-7i/TLR4/MyD88 signaling pathway. Pharmacol Res 111: 509-522, 2016.

25. Hei Z, Zhang A, Wei J, Gan X, Wang Y, Luo G and Li X: Lipopolysaccharide effects on the proliferation of NRK52E cells via alternations in gap-junction function. J Trauma Acute Care Surg 73: 67-72, 2012.

26. Livak KJ and Schmittgen TD: Analysis of relative gene expression data using real-time quantitative PCR and the $2(-\Delta \Delta \mathrm{C}(\mathrm{T}))$ method Methods 25: 402-408, 2001.

27. Schrier RW, Wang W, Poole B and Mitra A: Acute renal failure: definitions, diagnosis, pathogenesis, and therapy. J Clin Invest 114: 5-14, 2004.

28. Bihorac A, Baslanti TO, Cuenca AG, Hobson CE, Ang D, Efron PA, Maier RV, Moore FA and Moldawer LL: Acute kidney injury is associated with early cytokine changes after trauma. J Trauma Acute Care Surg 74: 1005-1013, 2013

29. Heyman SN, Khamaisi M, Rosen S and Rosenberger C: In vivo models of acute kidney injury. Drug Discov Today Dis Models 7: 51-56, 2010.

30. Kurozumi A, Goto Y, Matsushita R, Fukumoto I, Kato M, Nishikawa R, Sakamoto S, Enokida H, Nakagawa M, Ichikawa T, et al: Tumor-suppressive microRNA-223 inhibits cancer cell migration and invasion by targeting ITGA3/ITGB1 signaling in prostate cancer. Cancer Sci 107: 84-94, 2016.

31. Ichii O, Otsuka S, Sasaki N, Namiki Y, Hashimoto Y and Kon Y: Altered expression of microRNA miR-146a correlates with the development of chronic renal inflammation. Kidney Int 81: 280-292, 2012.

32. Yoon JH, Abdelmohsen $\mathrm{K}$ and Gorospe $\mathrm{M}$ : Functional interactions among microRNAs and long noncoding RNAs. Semin Cell Dev Biol 34: 9-14, 2014.

33. Cao Y, Xu R, Xu X, Zhou Y, Cui L and He X: Downregulation of lncRNA CASC2 by microRNA-21 increases the proliferation and migration of renal cell carcinoma cells. Mol Med Rep 14: 1019-1025, 2016.

34. Chiyomaru T, Fukuhara S, Saini S, Majid S, Deng G, Shahryary V, Chang I, Tanaka Y, Enokida H, Nakagawa M, et al: Long noncoding RNA HOTAIR is targeted and regulated by microRNA-141 in renal carcinoma cells. J Biol Chem 289: 12550-12565, 2014.

35. Wu Q, Han L, Yan W, Ji X, Han R, Yang J, Yuan J and Ni C: miR-489 inhibits silica-induced pulmonary fibrosis by targeting MyD88 and Smad3 and is negatively regulated by lncRNA CHRF. Sci Rep 6: 30921, 2016.

36. Gutschner T, Hämmerle M and Diederichs S: MALAT1 - a paradigm for long noncoding RNA function in cancer. J Mol Med (Berl) 91: 791-801, 2013. 